\title{
Congenital toxoplasmosis and DALYs in the Netherlands
}

\author{
LM Kortbeek ${ }^{1 /+}$, A Hofhuis ${ }^{2}$, CDM Nijhuis ${ }^{1}$, AH Havelaar ${ }^{3,4}$ \\ ${ }^{1}$ Laboratory for Infectious Diseases and Perinatal Screening, Centre for Infectious Disease Control Netherlands, National Institute for \\ Public Health and the Environment, PO Box 1, 3720 BA Bilthoven, the Netherlands ${ }^{2}$ Epidemiology and Surveillance Unit ${ }^{3}$ Laboratory for \\ Zoonoses and Environmental Microbiology ${ }^{4}$ Division of Veterinary Public Health, Institute for Risk Assessment Sciences, \\ Utrecht University, Utrecht, the Netherlands
}

The calculation of disability-adjusted life years (DALYs) enables public health policy makers to compare the burden of disease of a specific disease with that of other (infectious) diseases. The incidence of a disease is important for the calculation of DALYs. To estimate the incidence of congenital toxoplasmosis (CT), a random sample of 10,008 dried blood spot filter paper cards from babies born in 2006 in the Netherlands were tested for Toxoplasma gondii-specific IgM antibodies. Eighteen samples were confirmed as positive for IgM, resulting in an observed birth incidence of CT of 1.8 cases per 1,000 live-born children in 2006 and an adjusted incidence of 2.0 cases per 1,000. This means that 388 infected children were born in 2006. The most likely burden of disease is estimated to be 2,300 DALYs (range 820-6,710 DALYs). In the previous calculations, using data from a regional study from 1987, this estimate was 620 DALYs (range 220-1,900 DALYs). The incidence of CT in the Netherlands is much higher than previously reported; it is 10 times higher than in Denmark and 20 times higher than in Ireland, based on estimates obtained using the same methods. There is no screening program in the Netherlands; most children will be born asymptomatic and therefore will not be detected or treated.

Key words: toxoplasmosis - congenital - DALYs - burden of disease

Information on the burden of disease is essential in order to implement evidence-based public health policy. A methodology for the estimation of the burden of disease proposed by Murray et al. (1997a, b) takes into account the mortality, incidence (and/or prevalence) and sequelae associated with a disease, and expressed the burden in disability-adjusted life years (DALYs). Using DALYs, it is possible to compare the burden of a specific disease with that of other (infectious) diseases.

Congenital toxoplasmosis occurs when a woman is primarily infected with Toxoplasma during pregnancy. The parasite enters the foetal circulation by infection of the placenta. Infants born from mothers who acquire their infection in the first and second trimester more frequently show severe symptoms of congenital toxoplasmosis, or the infection may cause abortion/stillbirth. In contrast, the majority of children born to women who acquire their infection during the third trimester are born with the subclinical form of the infection (Dunn 1999, Gras 2005).

The incidence of a disease is of great importance in calculating DALYs. Data that were available in the Netherlands for congenital toxoplasmosis were old and biased. They were obtained from a well-informed study population in one region of the Netherlands in 1987. The seroprevalence in the Netherlands has since then decreased considerably, as it has in the rest of Europe, and there are differences between geographical regions (Kortbeek 2004).

+Corresponding author: titia.kortbeek@rivm.nl

Received 10 October 2008

Accepted 11 March 2009
To increase the reliability of our data set, we performed a study in neonates using dried blood spot cards. These cards became available after one year, but only for anonymous scientific research. Approximately 181,000206,000 babies are born alive each year in the Netherlands (Statistics Netherlands:www.cbs.nl). The estimated incidence derived from this study was used to recalculate the burden of disease as described by Havelaar et al. (2007). Havelaar et al. estimated the baseline disease burden of toxoplasmosis in the Netherlands at 620 DALYs, which is similar to the disease burden of salmonellosis and is mainly attributable to foetal loss and chorioretinitis.

\section{SUBJECTS, MATERIALS AND METHODS}

The different outcomes of infectious diseases can be combined into one single measure, the DALY, following the methodology proposed by Murray et al. (1997a, b): DALY $=$ YLL + YLD. YLL is the number of years of life lost due to mortality and YLD is the number of years lived with a disability, weighted by a factor between 0-1 for the severity of the disability. The YLL due to a specific disease in a specified population are calculated by the summation of all fatal cases $(d)$ due to the health outcomes $(l)$ of that specific disease, with each case multiplied by the expected individual life span $(e)$ at the age of death. Thus: $Y L L=\sum_{l} d_{l} \times e_{l}$.

The YLD are calculated by the summation over all cases $(n)$ and all health outcomes $(l)$ of the product of the duration of the illness $(t)$ and the severity weight $(w)$ of a specific disease: $Y L D=\sum_{l} n_{l} \times t_{l} \times w_{l}$.

The burden of disease attributable to one agent is obtained by cumulating the impact of all health outcomes associated with this agent. 
The health outcomes following a congenital Toxoplasma gondii infection were defined using an outcome tree. The disease burden is defined as the present expected sum of current and future DALYs resulting from all incident cases of disease in a specific time period, taking into account lifetime probabilities of transiting to each disease state. To estimate the incidence of the different health outcomes due to congenital toxoplasmosis, relevant studies in the literature were reviewed and the results were weighted for study size and pooled as previously described (Havelaar et al. 2007). Estimates on the duration of the adverse health outcomes were preferably derived from Dutch studies, but were supplemented as necessary with international studies. The expected life span of fatal cases occurring in 2004 was extracted from standard life tables as reported by Statistics Netherlands. Visual impairment occurs only in a fraction of children with chorioretinitis. The data from France and the United States published by Kodjikian et al. (2006) and Mets et al. (1996), respectively, were used as the low and high estimates; the most likely value was defined as the midpoint. To account for uncertainty, low values, most likely values and high values were used for the parameters.

The outcome tree consisted of the clinical manifestations of congenital toxoplasmosis: chorioretinitis, intracranial calcification, hydrocephalus, central nervous system (CNS) abnormalities and foetal death. Infants with sub-clinical infection in the first year of life may develop clinical signs and deficiencies later in life (mostly affecting the eyes) (Koppe et al. 1986, Binquet et al. 2003).

Study of the incidence of congenital toxoplasmosis in the Netherlands in 2006 - A random sample of 10,008 dried blood spot (DBS) cards was selected from children born in 2006, as described elsewhere in detail (A Hofhuis, unpublished observations). In short, an equal number of DBS cards was selected every other month starting in January 2006. The number of selected DBS cards varied between provinces of the Netherlands, as the sample size was weighted for the number of newborns within each province in 2006. For the cities of Amsterdam and Rotterdam, separate samples were collected. The DBS cards were tested anonymously and it was not possible to get information about the clinical situation of the children.

Eluates from the DBS cards were analysed and IgM antibodies were detected using the Wallac AutoDELFIA Neonatal Toxoplasma Screening kit. DBS cards with Toxoplasma-specific IgM antibodies above the cut-off (4 IU) in the screening analysis were confirmed by a modified IgM immunosorbent agglutination assay (ISAGA; BioMérieux, France) for Toxoplasma-specific IgM antibodies. The sensitivity and specificity of the methods used can be calculated from the results of the screening program in Denmark, in which the same methods were used and a specificity of $99.9 \%$ and sensitivity of $77 \%$ were reported (Lebech et al. 1999, Schmidt et al. 2006). For all samples that tested positive in the ISAGA assay, and for a random selection of 103 samples that tested negative, additional information was collected on the date of birth, date of heel puncture, birth weight, duration of pregnancy and degree of urbanisation.
The DALYs calculated for congenital toxoplasmosis were compared to the DALYs attributable to the foodborne agent Campylobacter spp., as reported by Mangen and Havelaar et al. (2005). To be able to compare the old Toxoplasma data from 1987-1988 to the new data, we needed to estimate the number of seronegative women in 2004.

Estimation of the number of seronegative women We used previously described methods (Havelaar et al. 2007). In short, the Toxoplasma Intervention Prevention (TIP) study was a prospective cohort study that started in 1987 and included 28,000 pregnant women in the SouthWest region of the Netherlands (Conyn 1991). Of 28,000 women, 15,170 (54\%) were seronegative and 12 newborn children had a congenital infection. Hence, the estimated incidence rate of congenital toxoplasmosis in 1987-1988 was 8.1 (4.7-12) per 10,000 live births from women who were seronegative at the start of their pregnancy.

The Pienter project was a cross-sectional seroprevalence study carried out in the Netherlands in 1996. In the childbearing age range of 15-50 years, the observed seroprevalence for toxoplasmosis increases linearly with age at a rate of $1.42 \%(90 \% \mathrm{CI}=1.21-1.63 \%)$ per year. By combining the age-specific seroprevalence rates from the Pienter study with the age distribution of pregnant women, we estimated that in 2004, approximately $129,000(67 \%, 90 \% \mathrm{CI}=61-72 \%)$ pregnant women were seronegative, hence at risk of transmitting toxoplasmosis to their unborn child.

Using the incidence rate of congenital toxoplasmosis of 0.81 per 1,000 live births from seronegative mothers, an incidence of 0.54 per 1,000 live births was calculated for the Netherlands in 2004.

\section{RESULTS}

Incidence of congenital toxoplasmosis - Thirty-two out of 10,008 samples were found to be positive in the Wallac IgM screen test and 18 of these 32 tested positive for Toxoplasma-specific IgM in the ISAGA assay, resulting in a birth incidence of 1.8 per 1,000 live-born children $(95 \%$ $\mathrm{CI}=1.1-2.8)$ in 2006 in the Netherlands. The incidence of congenital toxoplasmosis was not homogeneously spread throughout the country, as it appeared to be higher in the South-West than in the North-East of the Netherlands. No positive cases were detected in the North-East. Due to the sample size, the confidence intervals were too wide to show a statistically significant difference.

Using a specificity of $99.9 \%$ and sensitivity of $77 \%$, the true prevalence of congenital toxoplasmosis in the Netherlands was calculated to be two cases per 1,000 liveborn children. In 2004, there were 194,000 live births in the Netherlands, which means that 388 children were born with congenital toxoplasmosis $(95 \% \mathrm{CI}=213-543)$.

Incidence of health outcomes - We used data from the literature to estimate the frequency of foetal losses. The maximum estimate used was 4\% (Gratzl et al. 1998, Foulon et al. 1999, Binquet et al. 2003). Our most likely estimate is based on data collected by AFSSA, which estimated that, in France, 47 foetal losses due to toxoplasmosis occur every year among 2,676 (1.7\%) sero- 
converting mothers (Derouin et al. 2005). The minimum estimate was based on PALGA, the nationwide network and registry of histo and cytopatholgy in the Netherlands, which recorded an average 3 (1-10) foetal losses due to toxoplasmosis every year (Hofhuis et al. 2007).

The other health effects at birth from congenital toxoplasmosis for which the distribution was derived from the literature were: chorioretinitis, $13 \%(12-15 \%)$; intracranial calcification, $11 \%$ (8-12\%); hydrocephalus, $2 \%(1-3 \%)$; CNS abnormalities, $3 \%$ (1-6\%); and neonatal death, $0.7 \%$ (0.4-1.2\%) (Havelaar et al. 2007).

The most likely estimate for the probability of developing chorioretinitis later in life was based on the relatively large studies by Binquet et al. 2003 and Gras et al. 2005. In these studies, follow-up was performed up to 14 and 10 years, respectively, yielding a risk of developing chorioretinitis later in life of $2 \%$ per year. The reported rate in other studies varied between 1-3\%, which we applied as low and high estimates. Based on the study by Koppe et al. 1986, we assumed that the period in which children might develop chorioretinitis was at least 20 years. We used this time frame as our most likely estimate as well as our low estimate. As a high estimate, we arbitrarily doubled the period of risk to 40 years. The duration of the disorder is the life expectancy of the general population minus the mean age at which chorioretinitis develops $(79-10=69$ years $)$ (Table).

Calculation of the DALYS - To calculate the burden of disease, an incidence of 388 cases per year was used as the most likely estimate. The low and high estimates were obtained from the lower limit (1.1 case per 1,000, i.e. 213 cases) and upper limit ( 2.8 cases per 1,000 , i.e. 543 cases) of the $95 \%$ confidence interval, respectively.

The most likely burden of disease is 2,303 DALYs, with a range of 818-6,713 DALYs. In the previous calculations using the incidence from the TIP study, the estimate obtained was 620 DALYs (range 220-1,900). We compared the most likely burden of disease for congenital toxoplasmosis to the burden of Campylobacter as re- ported by Mangen et al. 2005. This comparison showed that, in this new calculation, Toxoplasma (Toxoplasma new data) has a greater burden than Campylobacter (1,200 DALYs, 95\% CI =900-1,600); the DALYs obtained in the new calculation were more than three times higher than the estimate using the old data (Toxoplasma old data). Campylobacter is generally considered to be the most important food-borne pathogen (Figure).

\section{DISCUSSION}

The goal of this study was to make it possible to compare the burden of disease of different (food-borne) pathogens with more accurate data. This information is essential for evidence-based public health policy. The incidence of congenital toxoplasmosis in the Netherlands was two per 1,000 live births, which is almost four times higher than the incidence found in the previous study performed 20 years earlier (TIP study 1987-1988). However, the study population in the 1987-1988 study was very well informed about the risk of congenital toxoplasmosis and a blood sample was taken from the pregnant women every three months. The study combined prevention with estimation of the incidence. The mothers in the present study received no specific information other than the normal leaflets for pregnant women. The disease burden in DALYs found in this study even exceeds the number that was calculated using the estimated incidence from the Pienter-study [1,200 DALYs per year (range 440-3,400)].

In this pilot study, major limitations were encountered due to the anonymous sampling and lack of information on the true positivity of the children. The calculation of the DALYs was partly limited because data on intra-uterine and perinatal mortality and information on the incidence and severity of complications and chronic sequelae were not available. The data on untreated children are very scarce and only available from small patient populations in a different timeframe (Koppe et al. 1986). Toxoplasmosis is not a reportable disease in the Neth-

TABLE

Incidence and disease burden of congenital toxoplasmosis in the Netherlands, 2004 (most likely estimates)

\begin{tabular}{|c|c|c|c|c|c|c|}
\hline & & cidenc & & & & \\
\hline & 388 & 213 & 543 & Duration & Severity & DALYs \\
\hline & Most likely & Low & High & (years) & weight & per year ${ }^{b}$ \\
\hline Fetal loss & & & & & & \\
\hline ( $\geq 24$ weeks) & 10.7 & 5.0 & 34.1 & 79 & 1.00 & 845 \\
\hline Clinical symptoms in first year & & & & & & \\
\hline Chorioretinitis & 50.3 & 25.6 & 81.4 & 79 & 0.08 & 318 \\
\hline Intracranial calcification & 42.7 & 17.0 & 65.2 & 79 & 0.01 & 34 \\
\hline Hydrocephalus & 7.7 & 2.13 & 16.3 & 79 & 0.36 & 219 \\
\hline CNS abnormalities & 11.6 & 2.13 & 32.6 & 79 & 0.36 & 330 \\
\hline Neonatal death & 2.7 & 0.85 & 6.51 & 79 & 1.00 & 213 \\
\hline Asymptomatic at birth & & & & & & \\
\hline Chorioretinitis later in life & 62.4 & 10.7 & 280.4 & 69 & 0.08 & 344 \\
\hline Total & & & & & & 2303 \\
\hline
\end{tabular}

$a$ : incidence in number of cases in 2004; $b$ : calculated as incidence (most likely) x duration x severity weight. 


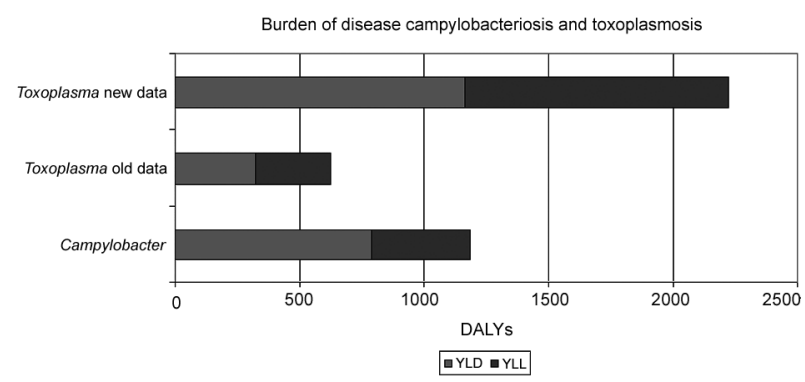

Burden of disease of Toxoplasma and Campylobacter in the Netherlands. YDL: years lived with disablily; YLL: years of life lost due to mortality.

erlands and there are no data available on the incidence of symptoms in different surveillance systems that can be used (Hofhuis \& Kortbeek 2006). We used European data as an alternative to national data. Data from Brazil were not used because the disease tends to be more often symptomatic at birth and ocular symptoms occur early in life in this region (Gilbert et al. 2008).

The incidence of congenital toxoplasmosis in the Netherlands is much higher than previously reported, with two infected children per 1,000 live births. This is 10 times higher than in Denmark (Schmidt et al. 2006) and 20 times higher than in Ireland (1 per 10,000) (P Mayne, personal communication), based on estimates obtained using the same methods. There is no screening program in the Netherlands and most children will be born asymptomatic and therefore will not be detected or treated.

Recalculation of the DALYs attributable to toxoplasmosis indicates that the burden of disease of congenital toxoplasmosis in the Netherlands is high; if combined with acquired toxoplasmosis, this burden will be even higher, indicating the importance of Toxoplasma as a food-borne pathogen.

\section{ACKNOWLEDGEMENTS}

To Yvonne van Duynhoven and Tineke Herremans (RIVM, Bilthoven, the Netherlands), for critically reviewing this paper and giving useful comments.

\section{REFERENCES}

Binquet C, Wallon M, Quantin C, Kodjikian L, Garweg J, Fleury J, Peyron F, Abrahamowicz M 2003. Prognostic factors for the long-term development of ocular lesions in 327 children with congenital toxoplasmosis. Epidemiol Infect 131: 1157-1168.

Conyn-Van Spaendonk MAE 1991. Prevention of congenital toxoplasmosis in the Netherlands, Bilthoven, RIVM, p. 51-80.

Derouin F, Bultel C, Roze S 2005. Toxoplasmose: état des connaissances et évaluation du risque lié a l'alimentation. Maison-Alfort, France: Agence Française de Securité Sanitaire des Aliments, p. 144-146.

Dunn D, Wallon M, Peyron F, Petersen E, Peckham C, Gilbert R 1999. Mother-to-child transmission of toxoplasmosis: risk estimates for clinical counselling. Lancet 353: 1829-1833.
Foulon W, Villena I, Stray-Pedersen B, Decoster A, Lappalainen M, Pinon JM, Jenum PA, Hedman K, Naessens A 1999. Treatment of toxoplasmosis during pregnancy: a multicenter study of impact on fetal transmission and children's sequelae at age 1 year. $\mathrm{Am} J$ Obstet Gynecol 180: 410-415.

Gilbert RE, Freeman K, Lago EG, Bahia-Oliveira LM, Tan HK, Wallon M, Buffolano W, Stanford MR, Petersen E, The European Multicentre Study on Congenital Toxoplasmosis (EMSCOT) 2008. Ocular sequelae of congenital toxoplasmosis in Brazil compared with Europe. PLoS Negl Trop Dis 2: e277.

Gras L, Wallon M, Pollak A, Cortina-Borja M, Evengard B, Hayde M, Petersen E, Gilbert R, European Multicenter Study on Congenital Toxoplasmosis 2005. Association between prenatal treatment and clinical manifestations of congenital toxoplasmosis in infancy: a cohort study in 13 European centres. Acta Paediatr 94: 1721-1731.

Gratzl R, Hayde M, Kohlhauser C, Hermon M, Burda G, Strobl W, Pollak A 1998. Follow-up of infants with congenital toxoplasmosis detected by polymerase chain reaction analysis of amniotic fluid. Eur J Clin Microbiol Infect Dis 17: 853-858.

Havelaar AH, Kemmeren JM, Kortbeek LM 2007. Disease burden of congenital toxoplasmosis. Clin Infect Dis 44: 1467-1474.

Hofhuis A, Kortbeek T 2006. Briefrapport Toxoplasma registratie in Nederland, Bilthoven, The Netherlands, p. 27.

Hofhuis A, van Duynhoven YTHP, Casparie MK, Kortbeek LM 2007. Intra-uteriene vruchtdood en perinatale sterfte door congenitale toxoplasmose in Nederland. Inf Bull 18: 344-347.

Kodjikian L, Wallon M, Fleury J, Denis P, Binquet C, Peyron F, Garweg JG 2006. Ocular manifestations in congenital toxoplasmosis. Graefes Arch Clin Exp Ophthalmol 244: 14-21.

Koppe JG, Loewer-Sieger DH, de Roever-Bonnet H 1986. Results of 20-year follow-up of congenital toxoplasmosis. Lancet 1: 254-256.

Kortbeek LM, De Melker HE, Veldhuijzen IK, Conyn-Van Spaendonck MAE 2004. Population-based Toxoplasma seroprevalence study in the Netherlands. Epidemiol Infect 132: 839-845.

Lebech M, Andersen O, Christensen NC, Hertel J, Nielsen HE, Peitersen B, Rechnitzer C, Larsen SO, Nørgaard-Pedersen B, Petersen E, Danish Congenital Toxoplasmosis Study Group 1999. Feasibility of neonatal screening for Toxoplasma infection in the absence of prenatal treatment. Lancet 353: 1834-1837.

Mangen MJJ, Havelaar AH, Bernsen RAJAM, van Koningsveld R, de Wit GA 2005. The costs of human Campylobacter infections and sequelae in the Netherlands - a DALY and cost-of-illness approach. Acta Agric Scand C Food Economics 2: 35-51.

Mets MB, Holfels E, Boyer KM, Swisher CN, Roizen N, Stein L, Stein M, Hopkins J, Withers S, Mack D, Luciano R, Patel D, Remington JS, Meier P, McLeod R 1996. Eye manifestations of congenital toxoplasmosis. Am J Ophthalmol 122: 309-324.

Murray CJ, Acharya AK 1997a. Understanding DALYs (disabilityadjusted life years). $J$ Health Econ 16: 703-730.

Murray CJ, Lopez AD 1997b. Global mortality, disability and the contribution of risk factors: globl burden of disease study. Lancet 349: 1436-1442.

Schmidt DR, Hogh B, Andersen O, Fuchs J, Fledelius H, Petersen E 2006. The national neonatal screening programme for congenital toxoplasmosis in Denmark: results from the initial four years, 1999-2002. Arch Dis Child 91: 661-665. 